Research Article

\title{
Indicators for the Compression and Stretching Characteristics of the HTF-Coordinate of WRF
}

\author{
Hailiang Zhang, ${ }^{1}$ Huoqing $\mathrm{Li}^{1}{ }^{1}$ and Shuiyong Fan $\mathbb{D}^{2}$ \\ ${ }^{1}$ Institute of Desert Meteorology, China Meteorological Administration, Urumqi 830002, China \\ ${ }^{2}$ Institute of Urban Meteorology, China Meteorological Administration, Beijing 100089, China \\ Correspondence should be addressed to Shuiyong Fan; syfan@ium.cn
}

Received 13 March 2020; Accepted 18 June 2020; Published 16 July 2020

Academic Editor: Marina Baldi

Copyright (c) 2020 Hailiang Zhang et al. This is an open access article distributed under the Creative Commons Attribution License, which permits unrestricted use, distribution, and reproduction in any medium, provided the original work is properly cited.

The HTF-coordinate is a feature that has been imported into the Weather Research and Forecasting (WRF) model version 4, and it is important to understand how it can be used efficiently. Indicators are needed to describe the characteristics of the distribution of the vertical coordinate and to evaluate the rationality of the configuration of the HTF-coordinate. Such indicators, including the maximum factor of compression (MFC), eta corresponding to the MFC, and the critical point of compression (CPC), were defined and calculated in this study. The indicators were also validated to efficiently and accurately describe the characteristics of the distribution of the vertical coordinate of the WRF model. They constituted the integrated system to resolve the problem of the configuration of the HTF-coordinate of WRF. The nonmonotonicity, compression, and stretching that were caused by the nonlinearity of the HTF-coordinate were also illustrated and validated.

\section{Introduction}

The terrain-following coordinate (TF-coordinate), which promotes the effective application of numerical weather prediction (NWP) models, has been used widely in various dynamic frameworks of such models [1-4]. However, it has certain defects, e.g., the use of terrain-following coordinates reduces the accuracy of the horizontal pressure gradient force (PGF) in regions of relative steep terrain [5] and divergent wind anomalies extend from the bottom to the top levels of the model and distort the terrain gravitational wave in steep terrain [6]. In high-resolution models, as the slope of the steep terrain becomes larger, the spurious disturbance caused by the numerical errors of the horizontal PGF will become more distinct [7], weakening the prediction performance of the high-resolution numerical model. To help mitigate these effects, various modifications to the TF-coordinate formulation have been introduced that more rapidly remove terrain influences in the coordinate surfaces with increasing height. The most successful and widely used approach is the hybrid terrain-following coordinate (HTFcoordinate) [8-10]. The HTF-coordinate smoothly transitions from the TF-coordinate at the surface to the purely isobaric coordinate (p-coordinate) at the higher levels of the model to diminish the effect of steep terrain [11-17]. Through the implementation of the hybrid coordinate, the WRF model significantly reduces small-scale spurious vertical velocities, particularly at upper levels and downstream of complex terrain $[18,19]$.

WRF is a regional numerical weather prediction model developed by the National Center of Atmospheric Research (NCAR) and is used worldwide. WRF v4.0 implements NCAR's HTF-coordinate using the Klemp cubic polynomial approach [20]. How to configure the parameters and take full advantage of the HTF-coordinate are important in the application of the hybrid coordinate system. Park et al. [21] deduced the necessary condition to maintain the monotonicity of the HTF-coordinate, which is an important result for the operational application of the HTF-coordinate. Indicators are also needed to describe the characteristics of the distribution of the vertical coordinate to choose the rational parameters of the HTF-coordinate of WRF. In this paper, the nonmonotonicity, compression, and stretching of the vertical coordinate were studied and the indicators of 
compression and stretching were defined, calculated, and validated. They constituted the indicator system to resolve the problem of the configuration of the HTF-coordinate.

Our paper is organized as follows. In Section 2, the basic contents and characteristics of the HTF-coordinate are introduced briefly. In Section 3, the nonmonotonicity of the HTF-coordinate is introduced and illustrated using idealized tests. In Section 4, the difference of the partial derivative of normalized $p d$ with respect to eta, the MFC, eta corresponding to the MFC, and the CPC of the HTF-coordinate are defined and calculated. They are then validated to allow the effective and accurate evaluations of the compression and stretching of the vertical coordinate in an idealized experiment. Section 5 presents some discussion. In Section 6 , the conclusions of the study are summarized.

\section{HTF-Coordinate Introduction}

This is a brief introduction to the basic contents of the HTFcoordinate to facilitate the calculations in the below sections.

2.1. TF-Coordinate. TF-coordinates are used in mesoscale numerical weather prediction models to simplify the processing of surface boundary conditions. The TF-coordinate in WRF has the form

$$
\begin{gathered}
\eta=\frac{p_{d}-p_{t}}{\mu}, \\
\mu=\left(p_{s}-p_{t}\right),
\end{gathered}
$$

where $p_{d}$ (noted as $p d$ ) is the hydrostatic component of the pressure of dry air and $p_{t}$ (noted as $p t$ ) and $p_{s}$ (noted as $p s$ ) are the hydrostatic top level pressure and the surface pressure for dry air, respectively. $\mu$ is the difference of hydrostatic pressure of dry air from the top level to the surface of the model; subscript $d$ indicates dry air. $\eta$ (noted as eta) indicates the vertical coordinate value of the model [18]. When $p_{d}=p_{s}$ and $\eta=1$, the vertical coordinate value is always 1 at the surface boundary.

\subsection{HTF-Coordinate. The WRF HTF-coordinate is given as}

$$
p_{d}=B(\eta)\left(p_{s}-p_{t}\right)+[\eta-B(\eta)]\left(p_{0}-p_{t}\right)+p_{t}
$$

where $p_{0}$ is the reference sea level pressure of air, $B(\eta)$ defines the relative weighting between the TF-coordinate and a purely isobaric coordinate, and $B(\eta)$ smoothly transitions from the TF-coordinate at the surface to the purely isobaric coordinate in the upper levels of the model [18-21]. $B(\eta)$ is defined in terms of a third-order polynomial:

$$
\begin{aligned}
B(\eta) & =c_{1}+c_{2} \eta+c_{3} \eta^{2}+c_{4} \eta^{3}, \\
B(1) & =1, \\
B_{\eta}(1) & =1, \\
B\left(\eta_{c}\right) & =0, \\
B_{\eta}\left(\eta_{c}\right) & =0 .
\end{aligned}
$$

Subject to the boundary conditions of equation (4),

$$
\begin{aligned}
& c_{1}=\frac{2 \eta_{c}^{2}}{\left(1-\eta_{c}\right)^{3}}, \\
& c_{2}=\frac{-\eta_{c}\left(4+\eta_{c}+\eta_{c}^{2}\right)}{\left(1-\eta_{c}\right)^{3}}, \\
& c_{3}=\frac{2\left(1+\eta_{c}+\eta_{c}^{2}\right)}{\left(1-\eta_{c}\right)^{3}}, c_{4}=\frac{-\left(1+\eta_{c}^{2}\right)}{\left(1-\eta_{c}\right)^{3}},
\end{aligned}
$$

where $\eta_{c}$ (noted as eta_c) is a user-defined constant that specifies where the vertical coordinate completely transitions from the TF-coordinate levels at low levels to purely isobaric coordinate levels aloft. The subscript $\eta$ represents the partial derivative with respect to eta. When $\eta=1$, the model applies the TF-coordinate at the surface; when $0 \leq \eta \leq \eta_{c}$, the model applies the $\mathrm{p}$-coordinate in the upper levels, and when $\eta_{c} \leq \eta \leq 1$, the transitions from TF-coordinate to $\mathrm{p}$-coordinate take place.

\section{The Nonmonotonicity of the HTF-Coordinate}

Although the HTF-coordinate reduces the errors in the PGF, it also generates other defects. For example, it cannot maintain the monotonic, continuous, and smooth one-toone mapping relationship between eta and $p_{d}$ at the lower levels of the model when $e t a \_c$ is set to excessively large values in regions of steep terrain, which causes the adjacent coordinate surfaces to intersect with each other, violating the monotonicity requirement of the coordinate.

3.1. HTF-Coordinate Numerical Experiment. Experiment 1 on the effect of the relationship between $p d$ and eta at different terrain altitudes and eta_c illustrates the nonmonotonicity of the vertical coordinate of the HTF-coordinate in WRF.

The configuration of the experiment is listed in Table 1. Four test cases were applied. From Figure 1, the following conclusions can be drawn:

(i) The nonlinearity relationship between eta and $p d$ at the lower level of the model became more significant when the topographic height or eta_c increased gradually.

(ii) The HF-, TF-, and p-coordinates were the same when the surface pressure of air equaled the referenced sea level pressure of air and the mapping relationship between eta and pd was completely linear.

(iii) The nonlinearity of the mapping relationship between eta and $p d$ is so significant that the nonmonotonicity of the vertical coordinate was achieved as $p_{s}=400 \mathrm{hPa}$ and eta_c $=0.5$ in the lower levels of the model. $p d$ with a smaller eta was greater than the $p d$ with a larger eta. This was unreasonable and resulted in the integral overflow of the numerical calculation of WRF. 
TABLE 1: Experimental parameters illustrating the nonmonotonicity of the HTF-coordinate.

\begin{tabular}{lcccc}
\hline Parameters & $p t(\mathrm{hPa})$ & $p 0(\mathrm{hPa})$ & $p s(\mathrm{hPa})$ & 1000 \\
$\eta_{c}$ \\
Case 1 & 10 & 1000 & 800 & $0.0-0.5$ \\
Case 2 & 10 & 1000 & 600 & $0.0-0.5$ \\
Case 3 & 10 & 1000 & 400 & $0.0-0.5$ \\
Case 4 & 10 & 1000 & $0.0-0.5$ \\
\hline
\end{tabular}

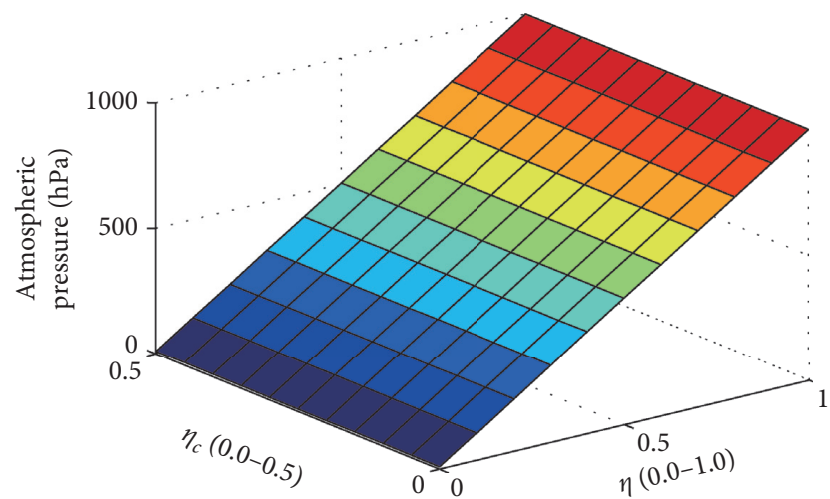

(a)

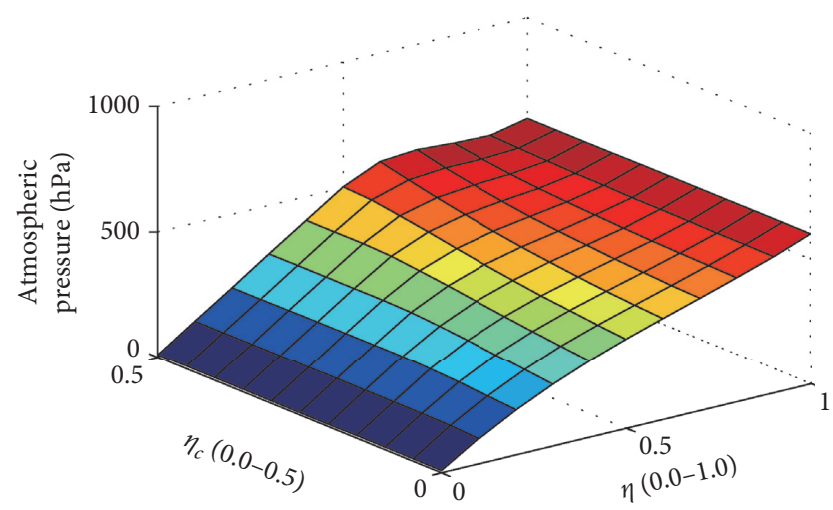

(c)

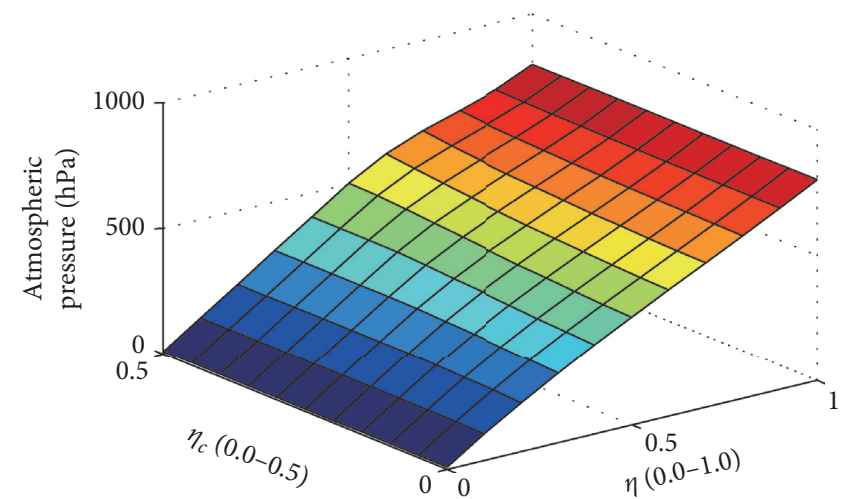

(b)

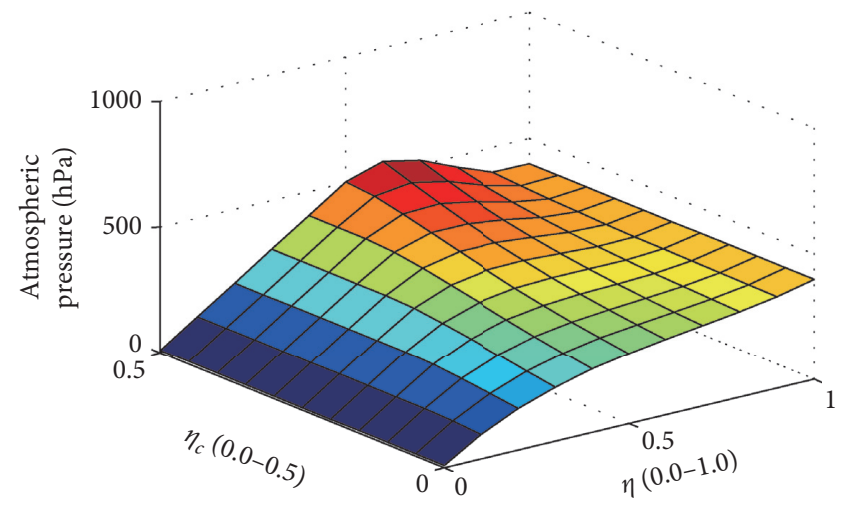

(d)

Figure 1: The impacts of different terrain heights and eta_c on the relationship between eta and $p d$ where (a) $p s=1000 \mathrm{hPa}$; $(\mathrm{b}) p s=800 \mathrm{hPa}$; (c) $p s=600 \mathrm{hPa}$; and (d) $p s=400 \mathrm{hPa}$.

Experiment 2. To further analyze the mapping relationship between $e t a$ and $p d$, an experiment with an ideal terrain was designed. The terrain was designed as a sine function:

$$
p_{s}=A \times\left|\sin \left(\frac{\pi}{10} x\right)\right|,
$$

where $A$ is the amplitude of the sine function and $p_{s}$ represents the height of the terrain. The parameters for Experiment 2 are listed in Table 2.

From Figure 2, the following conclusions can be drawn:

(i) The value of eta_c determines the height of the vertical coordinate above where the HTF-coordinate completely transitioned into the p-coordinate. The greater eta_c , the lower the level of the vertical coordinate of the model above where the HTFcoordinate transitioned into the p-coordinate. For example, the hydrostatic pressure of air was
TABLE 2: Experimental parameters to illustrate the terrain effect on the relationship between $e t a$ and $p d$.

\begin{tabular}{lcccc}
\hline Parameters & $p t(\mathrm{hPa})$ & $p 0(\mathrm{hPa})$ & $\mathrm{A}$ & $\eta_{c}$ \\
\hline Case 1 & 10 & 1000 & 800 & 0.2 \\
Case 2 & 10 & 1000 & 800 & 0.4 \\
Case 3 & 10 & 1000 & 500 & 0.2 \\
Case 4 & 10 & 1000 & 500 & 0.4 \\
\hline
\end{tabular}

approximately $200 \mathrm{hPa}$ at eta_c $=0.2$ or approximately $400 \mathrm{hPa}$ at eta_c $=0.4$ above where the HTFcoordinate completely transitioned into the p-coordinate.

(ii) The effect of the topography of the HTF-coordinate diminished with increasing height, and the mapping relationship between eta and $p d$ was completely linear when eta is less than eta_c. 


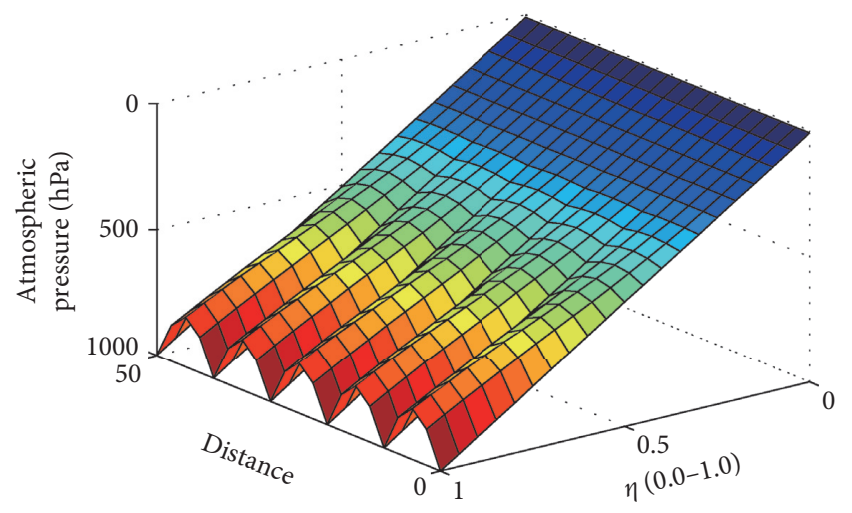

(a)

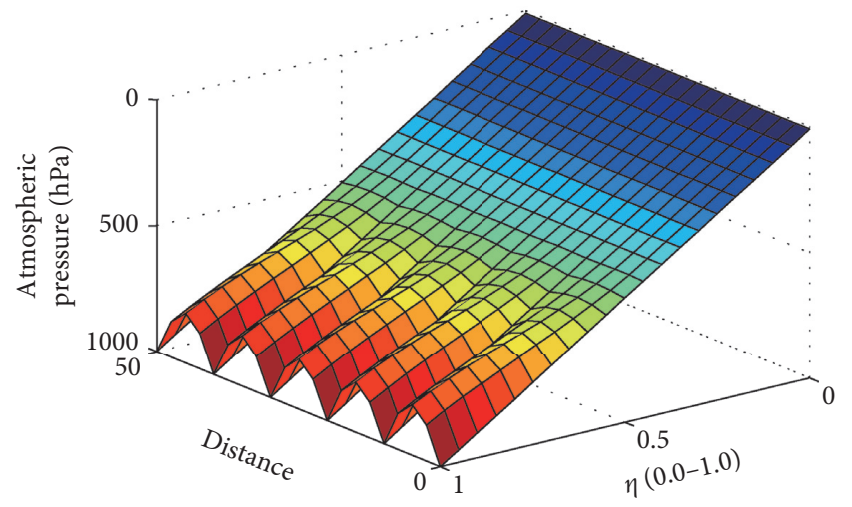

(c)

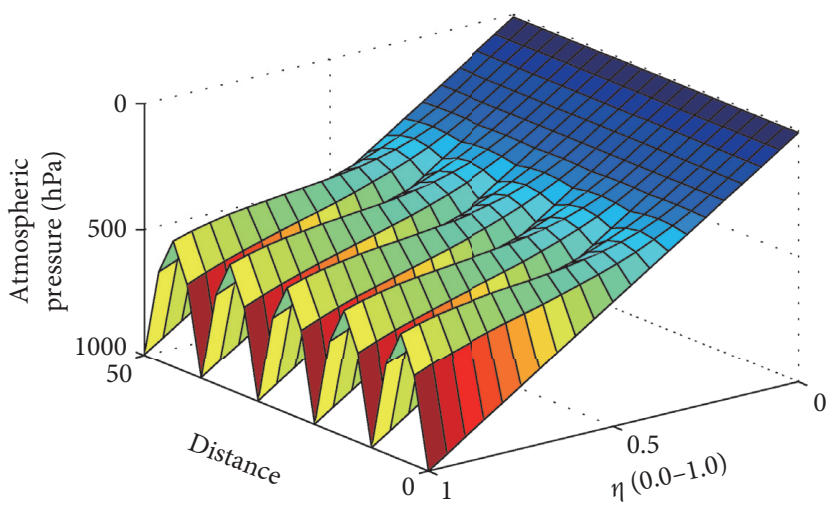

(b)

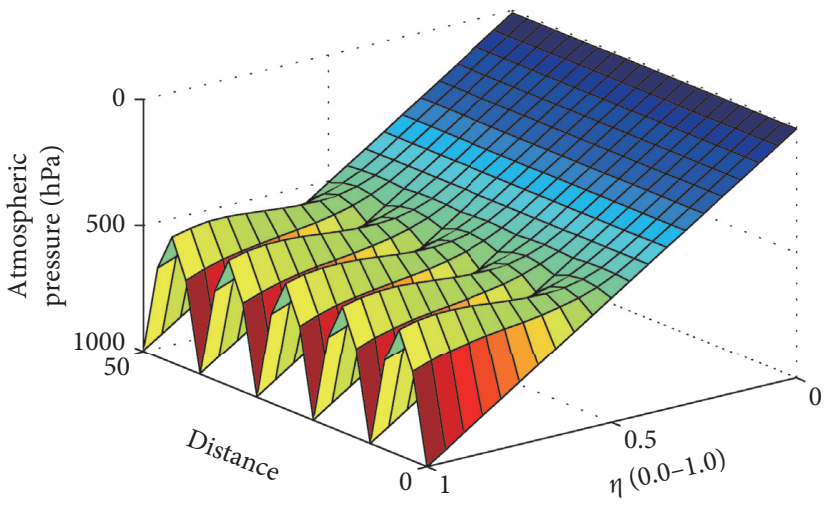

(d)

Figure 2: The impacts of topography on the relationship of eta and $p d$ with (a) a minimum surface pressure of $800 \mathrm{hPa}$, eta_c $=0.2$; (b) a minimum surface pressure of $800 \mathrm{hPa}$, eta_c $=0.4$; (c) a minimum surface pressure of $500 \mathrm{hPa}$, eta_c $=0.2$; and (d) a minimum surface pressure of $500 \mathrm{hPa}$, eta_c $=0.4$.

(iii) In theory, to reduce the PGF errors, eta_c should be set as large as possible; however, the nonmonotonicity of the HTF-coordinate limited the value range of eta_c.

The maximum value of eta_c should be studied to simulate WRF. To maintain the monotonic mapping relation between eta and $p d$, Park [21] found that $p_{s}$ and eta_c should conform to the following equations:

$$
\begin{aligned}
p_{s, \min }^{*} & =1-\left(1-p_{t}^{*}\right) \frac{3\left(\eta_{c}^{2}-1\right)}{-\left(\eta_{c}+2\right)^{2}}, \quad \eta_{c} \in(0,1), \\
p_{s, \min }^{*} & =\frac{p_{s, \min }}{p_{0}} \\
p_{t}^{*} & =\frac{p_{t}}{p_{0}}, \quad p_{s}^{*}>p_{s, \min }^{*}, \\
p_{s}^{*} & =\frac{p_{s}}{p_{0}} \\
p_{t}^{*} & =\frac{p_{t}}{p_{0}}
\end{aligned}
$$

where $p_{s, \text { min }}$ is the smallest allowable surface pressure for which the hybrid coordinate does not violate monotonicity. $p_{s, \text { min }}^{*}$ is the normalized $p_{s, \min }, p_{s}^{*}$ is the normalized hydrostatic surface pressure of the model, and $p_{t}^{*}$ is the normalized pressure at the top level of the model [21].

However, to ensure that the solution remains accurate and stable, it is important to configure the hybrid coordinate in a way that the anticipated minimum surface pressure $p_{s}^{*}$ is significantly greater than $p_{s, \min }^{*}$. According to (7), we created a relational table of $p_{t}^{*}, p_{s, \min }^{*}$, and eta_c as shown in Table 3.

\section{The Compression and Stretching of the HTF- Coordinate}

The nonlinear relationship between eta and $p d$ generated the compression and stretching of the vertical coordinate of the model. The approaches to measure the compression and stretching of the HTF-coordinate were defined, calculated, and validated to evaluate the compression and stretching of the vertical coordinate effectively and accurately in idealized experiments. 
TABLE 3: The minimum allowable surface pressure of air of the HTF-coordinate.

\begin{tabular}{lccccccccccc}
\hline Parameters & 0.0 & 0.1 & 0.2 & 0.3 & 0.4 & 0.5 & 0.6 & 0.7 & 0.8 & 0.9 & 1.0 \\
\hline 0.01 & 0.2575 & 0.3333 & 0.4109 & 0.4891 & 0.5669 & 0.6436 & 0.7188 & 0.7922 & 0.8636 & 0.9329 & 1 \\
0.02 & 0.265 & 0.34 & 0.4169 & 0.4943 & 0.5713 & 0.6472 & 0.7217 & 0.7943 & 0.865 & 0.9336 & 1 \\
0.03 & 0.2725 & 0.3467 & 0.4228 & 0.4994 & 0.5756 & 0.6508 & 0.7245 & 0.7964 & 0.8664 & 0.9343 & 1 \\
0.04 & 0.28 & 0.3535 & 0.4288 & 0.5046 & 0.58 & 0.6544 & 0.7273 & 0.7985 & 0.8678 & 0.9349 & 1 \\
0.05 & 0.2875 & 0.3602 & 0.4347 & 0.5097 & 0.5844 & 0.658 & 0.7302 & 0.8006 & 0.8691 & 0.9356 & 1 \\
0.06 & 0.295 & 0.3669 & 0.4407 & 0.5149 & 0.5888 & 0.6616 & 0.733 & 0.8027 & 0.8705 & 0.9363 & 1 \\
0.08 & 0.31 & 0.3804 & 0.4526 & 0.5252 & 0.5975 & 0.6688 & 0.7387 & 0.8069 & 0.8733 & 0.9376 & 1 \\
0.10 & 0.325 & 0.3939 & 0.4645 & 0.5355 & 0.6062 & 0.676 & 0.7444 & 0.8111 & 0.876 & 0.939 & 1 \\
0.20 & 0.4 & 0.4612 & 0.524 & 0.5871 & 0.65 & 0.712 & 0.7728 & 0.8321 & 0.8898 & 0.9458 & 1 \\
0.30 & 0.475 & 0.5286 & 0.5835 & 0.6388 & 0.6938 & 0.748 & 0.8012 & 0.8531 & 0.9036 & 0.9526 & 1 \\
\hline
\end{tabular}

The first row presents eta_c , the first column indicates $p_{t}^{*}$, and the $p_{s, \text { min }}^{*}$ values are listed in the table.

4.1. Compression and Stretching Experiment (Experiment 3). An experiment was designed and conducted to illustrate the compression and stretching of the vertical coordinate caused by the HTF-coordinate. The vertical coordinate of the model was divided into 50 even levels between 0 and 1 . The configuration of Experiment 3 is listed in Table 4.

As shown in Figure 3, pd was distributed evenly by the eta value in the case of the TF-coordinate. With the same configuration, the distribution of $p d$ was more concentrated at the lower levels and more decentralized at the higher levels of the model in Figures 3(b)-3(d). When eta_c was larger, the compression and stretching was more significant. At high levels of the model, the HTFcoordinate transitioned into a purely isobaric coordinate when $\eta \leq \eta_{c}$, which caused $p d$ to change almost linearly with eta.

4.2. Estimating the Compression and Stretching of the Vertical Coordinate. The compression and stretching of the vertical coordinate can be estimated using the difference of the partial derivative of the normalized $p d$ with respect to eta of the HTF-coordinate and that of the TF-coordinate. The rationality of the approach lies in the value of the partial derivative of the normalized $p d$ with respect to eta of the TF-coordinate being a constant as the $\mathrm{pt}^{*}$ is constant that can be standardized for the comparison. The difference represents the relative rate of change of normalized $p d$ with respect to eta between the TF- and the HTF-coordinate. If the difference is negative, this indicates compression of the vertical coordinate, and if the difference is positive, this indicates stretching. The greater the positive difference, the more significant the stretching. Conversely, the smaller the negative difference, the more significant the compression. The equation is derived as follows:

$$
\begin{aligned}
& \left(\frac{\partial p_{d}^{*}}{\partial \eta}\right)_{\text {sigma }}=\left(p_{s}^{*}-p_{t}^{*}\right) \\
& p_{s}^{*}=\frac{p_{s}}{p_{0}} \\
& p_{t}^{*}=\frac{p_{t}}{p_{0}} \\
& p_{d}^{*}=\frac{p_{d}}{p_{0}} \\
& \left(\frac{\partial p_{d}^{*}}{\partial \eta}\right)_{\text {hybrid }}=\frac{\left(\eta_{c}-\eta\right)\left(\eta_{c}-3 \eta-3 \eta_{c} \eta+\eta_{c}^{2}+4\right)}{\left(\eta_{c}-1\right)^{3}} \\
& \times\left(p_{s}^{*}-1\right)+\left(1-p_{t}^{*}\right), \quad \eta \in\left(\eta_{c}, 1\right), \\
& \left(\frac{\partial p_{d}^{*}}{\partial \eta}\right)_{\text {hybrid }}=1-p_{t}^{*}, \quad \eta \in\left(0, \eta_{c}\right), \\
& \left(\frac{\partial p_{d}^{*}}{\partial \eta}\right)_{\Delta}=\left(\frac{\partial p_{d}^{*}}{\partial \eta}\right)_{\text {hybrid }}-\left(\frac{\partial p_{d}^{*}}{\partial \eta}\right)_{\text {sigma }} \\
& =\frac{\left(\eta_{c}-\eta\right)\left(\eta_{c}-3 \eta-3 \eta_{c} \eta+\eta_{c}^{2}+4\right)}{\left(\eta_{c}-1\right)^{3}} \\
& \times\left(p_{s}^{*}-1\right)+\left(1-p_{s}^{*}\right), \quad \eta \in\left(\eta_{c}, 1\right), \\
& \left(\frac{\partial p_{d}^{*}}{\partial \eta}\right)_{\Delta}=1-p_{s}^{*}, \quad \eta \in\left(0, \eta_{c}\right)
\end{aligned}
$$

Equation (8) is the expression of the partial derivative of the normalized $p d$ with respect to eta of the TF-coordinate, and equation (9) is the expression of the HTF-coordinate. Equation (10) presents the difference of the partial derivative of the normalized $p d$ with respect to eta between the 
TABLE 4: Experimental parameters to illustrate and validate the compression and stretching of the vertical coordinate of the HTF-coordinate.

\begin{tabular}{lcccc}
\hline Parameters & $p t(\mathrm{hPa})$ & $p s(\mathrm{hPa})$ & $p 0(\mathrm{hPa})$ & $\eta_{c}$ \\
\hline Case 1 & 10 & 600 & - & 1000 \\
Case 2 & 10 & 600 & 1000 & 0.0 \\
Case 3 & 10 & 600 & 1000 & 0.2 \\
Case 4 & 10 & 600 & 0.4 \\
\hline
\end{tabular}

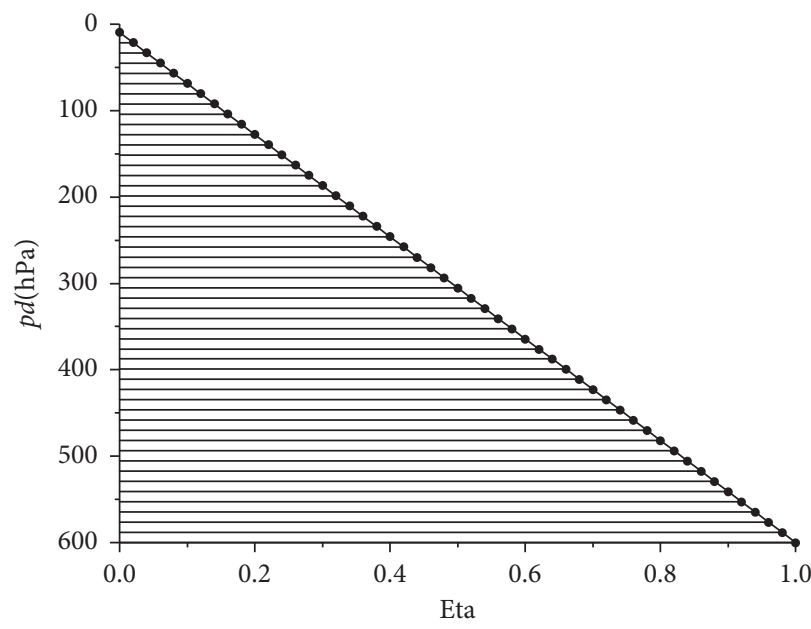

$\rightarrow$ TF-coordinate

(a)

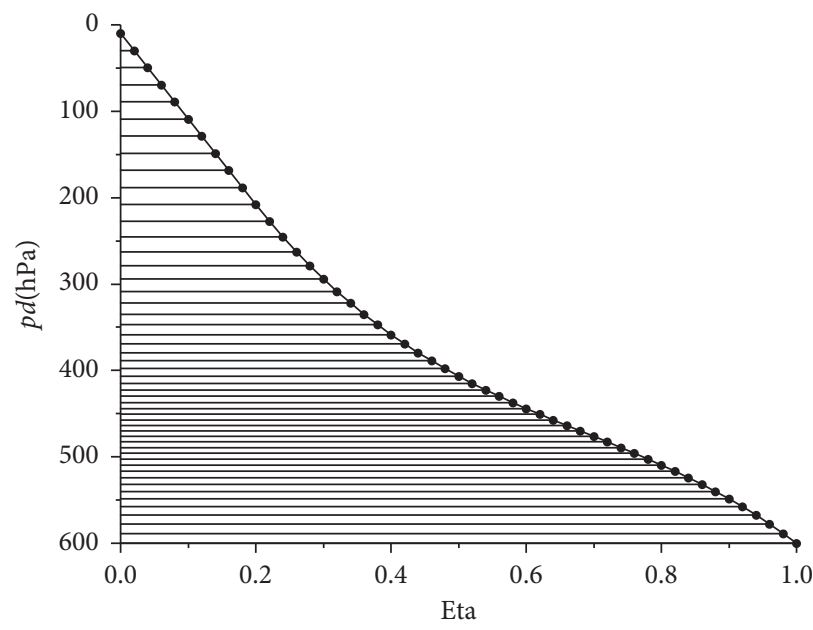

$\rightarrow$ eta_c $=0.2$

(c)

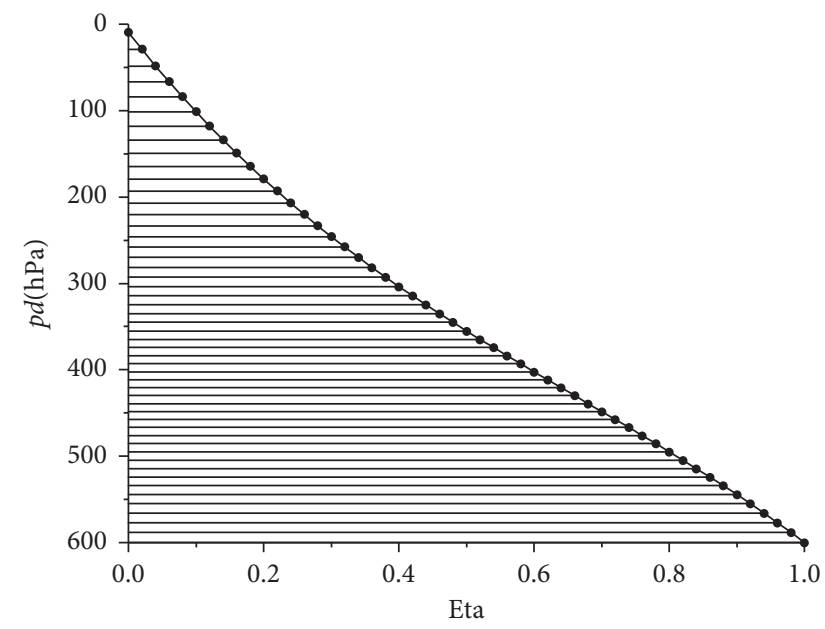

$\rightarrow$ eta_c $=0.0$

(b)

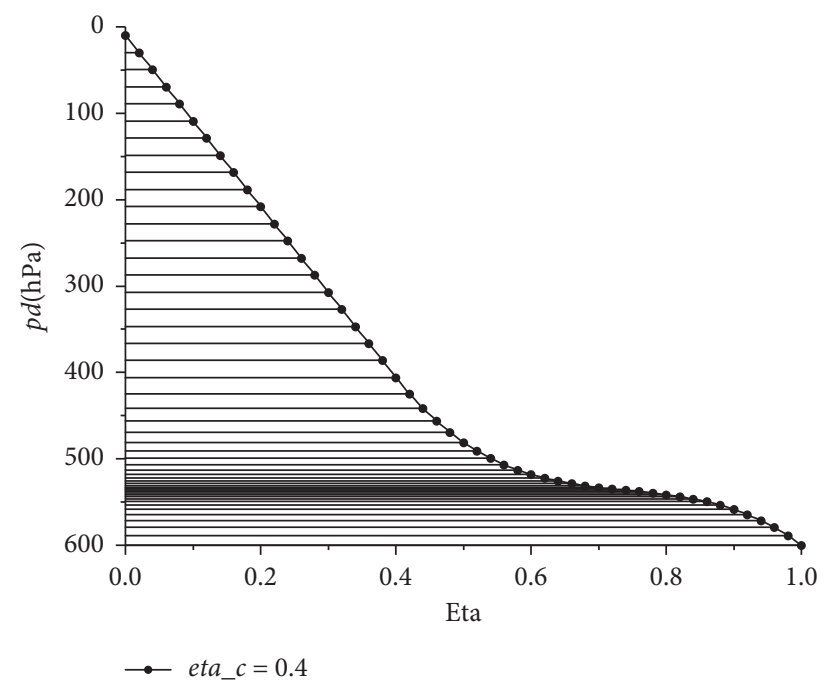

(d)

Figure 3: The compression and stretahing of the vertical coordinate of the HTF-coordinate. The horizontal coordinate represents eta of the model and the vertical coordinate represents $p d\left(p s=600 \mathrm{hPa}\right.$ and $p t=10 \mathrm{hPa}$ ). (a) TF-coordinate. (b) HTF-coordinate and $e t a \_c=0.0$. (c) HTF-coordinate and eta_c $=0.2$. (d) HTF-coordinate and eta_c $=0.4$.

HTF- and the TF-coordinate. Equation (10) shows that the difference of the partial derivative of the normalized $p d$ with respect to eta is only relevant to eta_c and the normalized surface pressure. Equations (9) and (10) are piecewise functions because the HTF-coordinate completely transitions into the purely isobaric coordinate when $\eta \in\left(0, \eta_{c}\right)$.

From Figure 4, the following conclusions can be drawn: (i) The difference of the partial derivative of the normalized $p d$ with respect to eta can estimate the compression and stretching of the vertical coordinate effectively and has a point with a minimum negative value that indicates the position where the compression of the vertical coordinate is most significant. The difference will be a constant when 


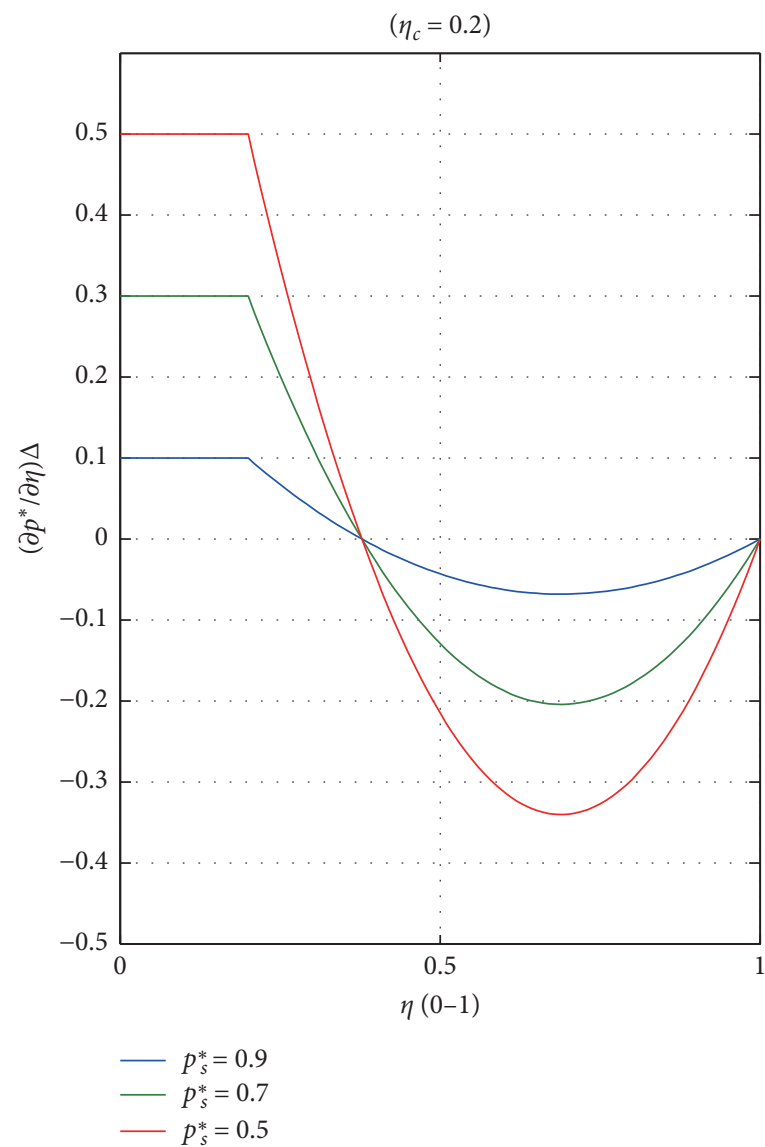

(a)

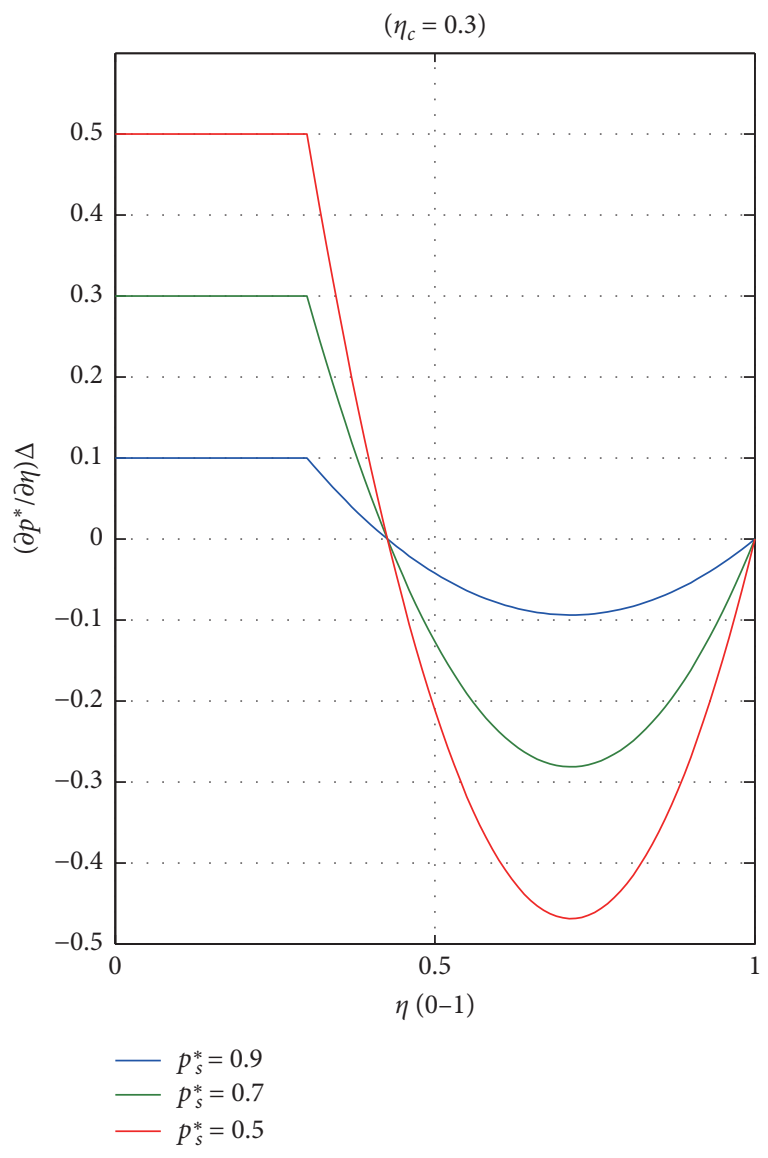

(b)

FIgURE 4: The relationship between the difference of the partial derivative of the normalized $p d$ with respect to eta and eta under different conditions of $p s^{*}$ and $e t a \_c$ : (a) eta_c $=0.2$ and (b) $e t a \_c=0.3$. $p t^{*}$ equals 0.01 .

eta is less than eta_c because $B(\eta)=0$ when eta is less than eta_c.

(ii) The absolute value of difference decreases with increasing normalized surface pressure and the gradually decreasing eta_c of the model. The small absolute value of difference indicates that the compression and stretching are moderate.

(iii) The eta value of the points where the difference equals zero can be calculated using equation (10) by setting the partial derivative of the normalized $p d$ with respect to eta to zero. The eta value of the points is only relevant to eta_c and where there is no compression or stretching of the vertical coordinate.

$$
\begin{aligned}
\left(\frac{\partial p_{d}^{*}}{\partial \eta}\right)_{\Delta} & =0 \\
\eta & =\frac{4 \eta_{c}^{2}+\eta_{c}+1}{3 \eta_{c}+3}, \quad \eta=1 .
\end{aligned}
$$

4.3. MFC of the HTF-Coordinate. It is reasonable to estimate the compression using the minimum difference of the partial derivative of the normalized $p d$ with respect to eta. The MFC is defined in terms of the absolute value of the minimum difference and is calculated as follows:

$$
\begin{aligned}
\left(\frac{\partial p_{d}^{*}}{\partial \eta}\right)_{\Delta, \max } & =\left|\frac{-\left(2 \eta_{c}+1\right)^{2}\left(p_{s}^{*}-1\right)}{3\left(\eta_{c}^{2}-1\right)}\right|, \\
& \eta_{c} \in(0,1), p_{s}^{*} \in(0,1) .
\end{aligned}
$$

As shown in equation (12), the MFC a positive value. The compression of the vertical coordinate is more significant with an increasing MFC. Note that eta_c should not be set to zero as this would result in the MFC being infinity.

MFC is only relevant to the normalized surface pressure and eta_c. From Figure 5, the following conclusions can be drawn:

(i) MFC increases with decreasing normalized surface pressure and increasing eta_c of the model.

(ii) MFC approaches infinity when eta_c approaches 1 .

(iii) MFC is zero when the normalized surface pressure is set to 1 , which means that no compression or stretching of the vertical coordinate occurs.

The MFC of the vertical coordinate was calculated based on equation (12) as shown in Table 5. 


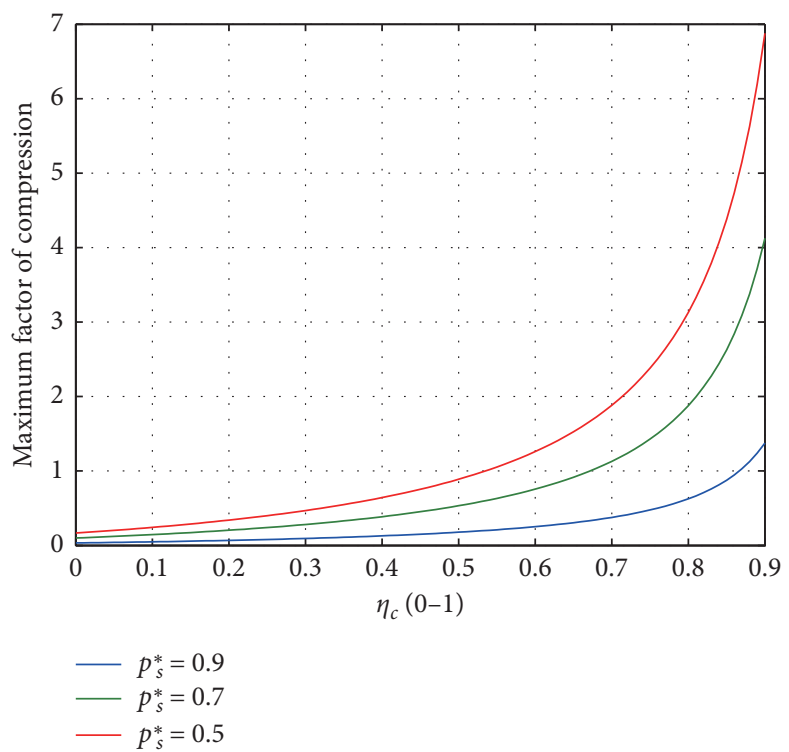

FIGURE 5: The relationship between MFC and eta_c. $p s^{*}=0.9,0.7$, and 0.5 , respectively.

TABLE 5: The MFC of the vertical coordinate.

\begin{tabular}{lccccccccccc}
\hline Parameters & 0.0 & 0.1 & 0.2 & 0.3 & 0.4 & 0.5 & 0.6 & 0.7 & 0.8 & 0.9 \\
\hline 1 & 0 & 0 & 0 & 0 & 0 & 0 & 0 & 0 & 0 & 0 \\
0.95 & 0.0167 & 0.0242 & 0.034 & 0.0469 & 0.0643 & 0.0889 & 0.126 & 0.1882 & 0.313 & 0.6877 \\
0.9 & 0.0333 & 0.0485 & 0.0681 & 0.0938 & 0.1286 & 0.1778 & 0.2521 & 0.3765 & 0.6259 & 1.3754 \\
0.8 & 0.0667 & 0.097 & 0.1361 & 0.1875 & 0.2571 & 0.3556 & 0.5042 & 0.7529 & 1.2519 & 2.7509 \\
0.7 & 0.1 & 0.1455 & 0.2042 & 0.2813 & 0.3857 & 0.5333 & 0.7563 & 1.1294 & 1.8778 & 4.1263 \\
0.6 & 0.1333 & 0.1939 & 0.2722 & 0.3751 & 0.5143 & 0.7111 & 1.0083 & 1.5059 & 2.5037 & 5.5018 \\
0.5 & 0.1667 & 0.2424 & 0.3403 & 0.4689 & 0.6429 & 0.8889 & 1.2604 & 1.8824 & 3.1296 & 6.8772 \\
0.4 & 0.2 & 0.2909 & 0.4083 & 0.5626 & 0.7714 & 1.0667 & 1.5125 & 2.2588 & 3.7556 & 8.2526 \\
0.3 & 0.2333 & 0.3394 & 0.4764 & 0.6564 & 0.9 & 1.2444 & 1.7646 & 2.6353 & 4.3815 & 9.6281 \\
\hline
\end{tabular}

The first row lists eta_c, the first column is $p_{s}^{*}$, and the table lists the values for MFC.

4.4. Eta Corresponding to the MFC. Eta corresponding to the MFC indicates eta where the compression of the vertical coordinate is most significant and is calculated using the following equation:

$$
\eta=\frac{2 \eta_{c}}{3}+\frac{2}{3\left(\eta_{c}+1\right)}, \quad \eta_{c} \in(0,1)
$$

Eta is only relevant to eta_c. The relationship between eta corresponding to the MFC and eta_c is shown in Figure 6.

Based on Figure 6, eta corresponding to the MFC increases with increasing $e t a \_c$ and is always less than $e t a \_c$. The values of eta corresponding to the MFC were calculated and are listed in Table 6.

4.5. CPC of the HTF-Coordinate. The vertical coordinate of the HTF-coordinate is divided into two intervals. One

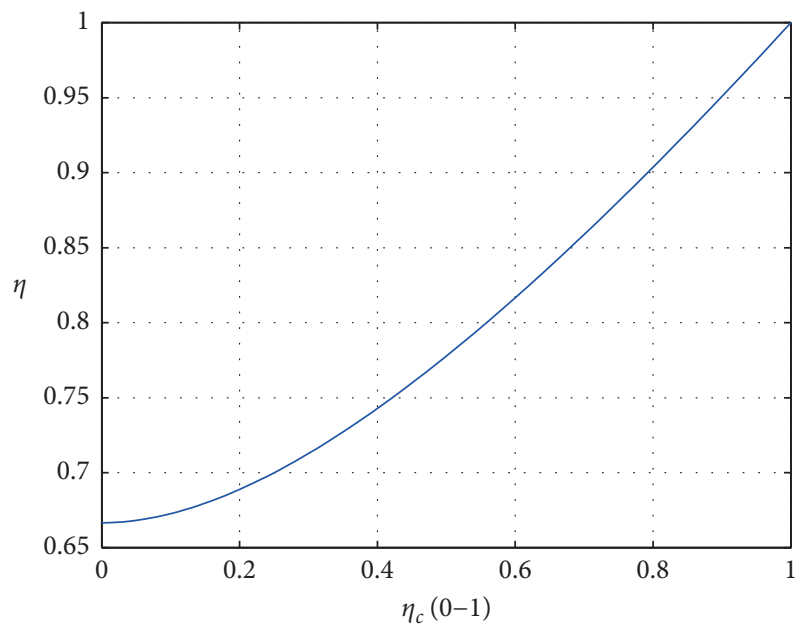

FIGURE 6: Eta corresponding to the MFC with eta_c. 
TABLE 6: Eta corresponding to the MFC of the vertical coordinate.

\begin{tabular}{|c|c|c|c|c|c|c|c|c|c|c|c|}
\hline$\eta_{c}$ & 0.0 & 0.1 & 0.2 & 0.3 & 0.4 & 0.5 & 0.6 & 0.7 & 0.8 & 0.9 & 1.0 \\
\hline$\eta$ & 0.6667 & 0.6727 & 0.6889 & 0.7128 & 0.7429 & 0.7778 & 0.8167 & 0.8588 & 0.9037 & 0.9509 & 1 \\
\hline
\end{tabular}

TABle 7: The eta value of the CPC of the vertical coordinate.

\begin{tabular}{cccccccccccc}
\hline$\eta_{c}$ & 0.0 & 0.1 & 0.2 & 0.3 & 0.4 & 0.5 & 0.6 & 0.7 & 0.8 & 0.9 & 1.0 \\
\hline$\eta$ & 0.3333 & 0.3455 & 0.3778 & 0.4256 & 0.4857 & 0.5556 & 0.6333 & 0.7176 & 0.8074 & 0.9018 & 1 \\
\hline
\end{tabular}

interval covers the lower levels of the vertical coordinate where the mapping relation between $p d$ and eta is compressed. The other covers the higher levels of the vertical coordinate where the mapping relation is stretched, and the CPC indicates the location where the transition occurs.

According to (11), when $\eta>\left(4 \eta_{c}^{2}+\eta_{c}+1 / 3 \eta_{c}+3\right)$, the mapping relation of $p d$ and eta is compressed, and when $\eta<\left(4 \eta_{c}^{2}+\eta_{c}+1 / 3 \eta_{c}+3\right)$, the mapping relation of $p d$ and eta is stretched. This is determined based on the eta_c.

To be able to conveniently look up the critical point, the CPC is listed in Table 7.

As shown in Figure 7, the vertical coordinate is compressed in the region above the curve and stretched in the region under the curve. When eta_c increases, the compressed region gradually decreases and the stretched region gradually increases accordingly.

4.6. Validation of the Compression of HTF-Coordinate Theory. The compression theory was validated using parameters from Experiment 3, where the normalized surface pressure is 0.6 and eta_c is set as $0.0,0.2$, and 0.4 , respectively. The MFC, eta corresponding to the MFC, and the CPC are calculated and listed in Table 8.

Based on Table 8 and Figure 3, the MFC is 0.5143 when eta_c $=0.4$, which is reasonable because the compression of the vertical coordinate is most significant in Figure 3(d). Eta corresponding to the MFC is 0.7429 when eta_c $=0.4$, which agrees well with Figure $3(\mathrm{~d})$. The CPC is 0.4857 when eta_c $=0.4$, which indicates the location of the completely transition in Figure 3(d). According to the validation, the compression theory of the HTF-coordinate can be used to efficiently estimate the compression and stretching of the vertical coordinate of the HTF-coordinate.

\section{Discussion}

Although WRF is used widely and the HTF-coordinate is imported into WRF v4.0, the accurate configuration of the hybrid coordinate is not explicitly noted in the WRF guide. Park et al. [21] systematically studied the nonlinearity of the HTF-coordinate of WRF in 2019 and stated the necessary conditions to maintain the linearity of the HTF-coordinate, which is important for the application of the HTF-coordinate. However, more indicators are needed to reveal the characteristics of the distribution of the vertical coordinate of the HTF-coordinate and to understand where the vertical coordinate is mainly compressed, where the compression

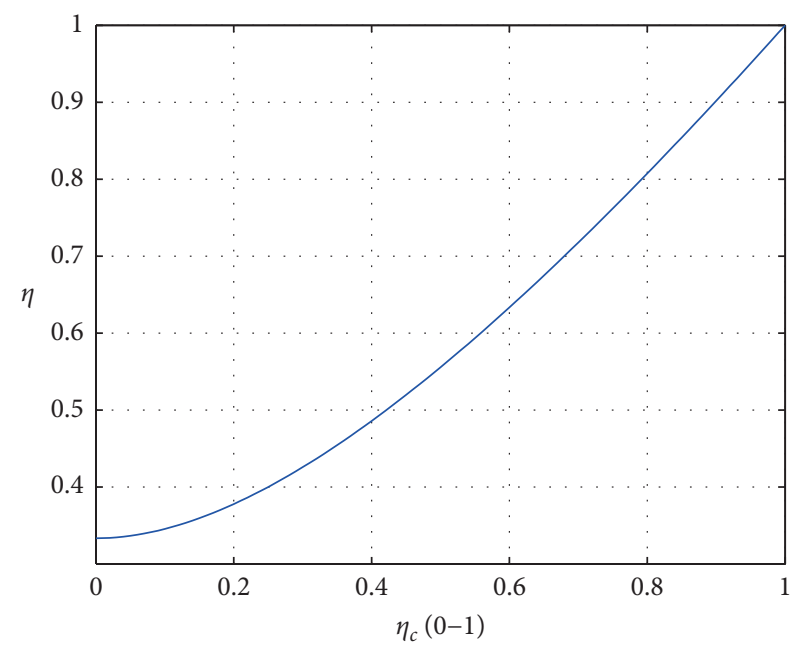

FIgURE 7: The CPC of the HTF-coordinate with eta_c.

TABLE 8: The compression indicators of different eta_c $(0.0,0.2,0.4)$ with $p s^{*}=0.6$.

\begin{tabular}{lccc}
\hline$\eta_{c}$ & MFC & $\eta$ responding to the MFC & CPC \\
\hline 0.0 & 0.1333 & 0.6667 & 0.3333 \\
0.2 & 0.2722 & 0.6889 & 0.3778 \\
0.4 & 0.5143 & 0.7429 & 0.4857 \\
\hline
\end{tabular}

transitions to stretching, and the degree of the compression that is important for the operational application of WRF.

The difference of the partial derivative of the normalized $p d$ with respect to eta, the MFC, eta corresponding to the MFC, and the CPC of the HTF-coordinate are defined, calculated, and validated to evaluate the compression and stretching of the vertical coordinate effectively and accurately. However, further analysis is needed to understand which distribution of the vertical coordinate is best suited for WRF to reduce the numerical errors of PGF over steep terrains.

\section{Conclusions}

(i) The nonmonotonicity of the HTF-coordinate indicated that the mapping relationship between eta and $p d$ was not monotonic, which was validated by the results of Experiments 1 and 2. This potentially caused the adjacent coordinate surfaces to intersect with each other, which violated the monotonicity 
requirement of the vertical coordinate and resulted in the integral overflow of the numerical computation. The nonmonotonicity was relevant to the value of eta_c, $p_{t}$, and $p_{s}$.

(ii) The compression and stretching of the vertical coordinate were caused by the nonlinearity of the HTF-coordinate. The compression and stretching were increasingly significant with increasing eta_c and decreasing $p_{s}^{*}$.

(iii) The difference of the partial derivative of the normalized $p d$ with respect to eta indicated the degree of compression and stretching of the vertical coordinate effectively and accurately.

(iv) The MFC of the HTF-coordinate was an effective indicator to estimate the compression of the vertical coordinate and was only relevant to the normalized surface pressure and eta_c. The compression of the vertical coordinate was more significant with an increasing MFC.

(v) Eta corresponding to the MFC indicated the value of eta where the compression of the vertical coordinate was most significant and was only relevant to eta_c. Eta corresponding to the MFC increased with increasing eta_c and was always less than eta_c.

(vi) The vertical coordinate was divided into two intervals by the CPC: one interval covered the lower levels of the vertical coordinate where the mapping relation between $p d$ and eta was compressed and the other covered the higher levels of the vertical coordinate where the mapping relation was stretched. When eta_c increased, the compressed region was gradually reduced, and the stretched region gradually increased accordingly.

\section{Data Availability}

The data used to support the findings of this study are included within the article.

\section{Conflicts of Interest}

The authors declare that they have no conflicts of interest.

\section{Authors' Contributions}

Hailiang Zhang contributed to original draft preparation, review and editing, and methodology. Huoqing Li was responsible for formal analysis and investigation. Shuiyong Fan conceptualized the study. Hailiang Zhang and Huoqing Li validated the study and were responsible for software. All authors read and agreed to the published version of the manuscript.

\section{Acknowledgments}

This research was funded by the National Key Research and Development Program of China (grant no. 2018YFC1507100), the Central Scientific Research Institute of the Public Basic
Scientific Research Business Professional (grant no. IDM2017001), the National Natural Science Foundation of China (grant no. 41875023), the Research Foundation of China Desert Meteorology (grant no. sqj2018017), the National Department of Public Benefit (Meteorology) Research Foundation (grant no. GYHY201306066), and the Central Asia Atmospheric Research Foundation (grant no. CASS201711).

\section{References}

[1] N. A. Phillips, "A coordinate system having some special advantages for numerical forecasting," Journal of Meteorology, vol. 14, no. 2, pp. 184-185, 1957.

[2] R. Bleck, "An oceanic general circulation model framed in hybrid isopycnic-Cartesian coordinates," Ocean Modelling, vol. 4, no. 1, pp. 55-88, 2002.

[3] T. Davies, M. J. P. Cullen, A. J. Malcolm et al., "A new dynamical core for the met office's global and regional modelling of the atmosphere," Journal of the Atmospheric Sciences, Applied Meteorology and Physical Oceanography, vol. 131, pp. 1759-1782, 2010.

[4] W. C. Skamarock, J. B. Klemp, M. G. Duda et al., "A multiscale nonhydrostatic atmospheric model using centroidal voronoi tesselations and C-grid staggering," Monthly Weather Review, vol. 240, pp. 3090-3105, 2011.

[5] F. Mesinger and Z. I. Janjic, "Problems and numerical methods of the incorporation of mountains in atmospheric models," Lectures in Applied Mathematics, vol. 22, pp. 81-120, 1985.

[6] S. D. Eckermann, J. P. McCormack, J. Ma, T. F. Hogan, and K. A. Zawdie, "Stratospheric analysis and forecast errors using hybrid and sigma coordinates," Monthly Weather Review, vol. 142, no. 1, pp. 476-485, 2014.

[7] J. Steppeler, R. Hess, U. Schttler, and L. Bonaventura, "Review of numerical methods for nonhydrostatic weather prediction models," Meteorology and Atmospheric Physics, vol. 82, no. 14, pp. 287-301, 2003.

[8] A. J. Simmons and D. M. Burridge, "An energy and angularmomentum conserving vertical finite-difference scheme and hybrid vertical coordinates," Monthly Weather Review, vol. 109, no. 4, pp. 758-766, 1981.

[9] J. B. Klemp, "A terrain-following coordinate with smoothed coordinate surfaces," Monthly Weather Review, vol. 139, no. 7, pp. 2163-2169, 2011.

[10] F. Mesinger, S. C. Chou, J. L. Gomes et al., "An upgraded version of the eta model," Meteorology and Atmospheric Physics, vol. 116, no. 3-4, pp. 63-79, 2012.

[11] Y. Li, B. Wang, D. Wang, J. Li, and L. Dong, "An orthogonal terrain-following coordinate and its preliminary tests using 2D idealized advection experiments," Geoscientific Model Development, vol. 7, no. 4, pp. 1767-1778, 2014.

[12] J. Li, Y. Li, B. Wang, and X. Zou, "Advection errors in an orthogonal terrain-following coordinate: idealized experiments," Chinese Science Bulletin, vol. 60, no. 32, pp. 31443152, 2015.

[13] S. Eckermann, "Hybrid $\sigma$-p coordinate choices for a global model," Monthly Weather Review, vol. 137, no. 1, pp. 224-245, 2009.

[14] A. J. Simmons and R. Strüfing, "Numerical forecasts of stratospheric warming events using a model with a hybrid vertical coordinate," Quarterly Journal of the Royal Meteorological Society, vol. 109, no. 459, pp. 81-111, 1983. 
[15] X. Zou, Y. Li, J. Li, and B. Wang, "Advection errors in an orthogonal terrain-following coordinate: idealized 2-D experiments using steep terrains," Atmospheric Science Letters, vol. 17, no. 3, pp. 243-250, 2016.

[16] S.-H. Park, W. C. Skamarock, J. B. Klemp, L. D. Fowler, and M. G. Duda, "Evaluation of global atmospheric solvers using extensions of the Jablonowski and Williamson Baroclinic Wave Test case," Monthly Weather Review, vol. 141, no. 9, pp. 3116-3129, 2013.

[17] C. Li, D. Chen, X. Li, and J. Hu, "Effects of terrain-following vertical coordinates on high-resolution NWP simulations," Journal of Meteorological Research, vol. 29, no. 3, pp. 432-445, 2015.

[18] J.-H. Kim, R. D. Sharman, S. G. Benjamin, J. M. Brown, S.-H. Park, and J. B. Klemp, "Improvement of mountain-wave turbulence forecasts in NOAA's rapid refresh (RAP) model with the hybrid vertical coordinate system," Weather and Forecasting, vol. 34, no. 3, pp. 773-780, 2019.

[19] J. Beck, J. Brown, J. Dudhia et al., "An evaluation of a hybrid, terrain-following vertical coordinate in the WRF-based RAP and HRRR models," Weather and Forecasting, vol. 35, pp. 1081-1096, 2020.

[20] W. C. Skamarock, B. Klemp, J. Dudhia et al., A Description of the Advanced Research WRF Version 4, NCAR Tech, Boulder, CO, USA, 2019.

[21] S.-H. Park, J. B. Klemp, and J.-H. Kim, "Hybrid mass coordinate in WRF-ARW and its impact on upper-level turbulence forecasting," Monthly Weather Review, vol. 147, no. 3, pp. 971-985, 2019. 